\title{
Deeply Virtual Pseudoscalar Meson Production at Jefferson Lab and Transversity GPDs
}

\author{
Valery Kubarovsky \\ Thomas Jefferson National Accelerator Facility \\ Newport News, VA 23606, USA \\ vpk@jlab.org \\ and the CLAS Collaboration
}

Published 29 February 2016

\begin{abstract}
The cross section of the exclusive $\pi^{0}$ and $\eta$ electroproduction reaction $e p \rightarrow e^{\prime} p^{\prime} \pi^{0} / \eta$ was measured at Jefferson Lab with a $5.75-\mathrm{GeV}$ electron beam and the CLAS detector. Differential cross sections $d^{4} \sigma / d t d Q^{2} d x_{B} d \phi_{\pi}$ and structure functions $\sigma_{T}+\epsilon \sigma_{L}, \sigma_{T T}$ and $\sigma_{L T}$ as functions of $t$ were obtained over a wide range of $Q^{2}$ and $x_{B}$. The data are compared with the GPD based theoretical models. Analyses find that a large dominance of transverse processes is necessary to explain the experimental results. Generalized form factors of the transversity GPDs $\left\langle H_{T}\right\rangle^{\pi, \eta}$ and $\left\langle\bar{E}_{T}\right\rangle^{\pi, \eta}$ were directly extracted from the experimental observables for the first time. It was found that GPD $\bar{E}_{T}$ dominates in pseudoscalar meson production. The combined $\pi^{0}$ and $\eta$ data opens the way for the flavor decomposition of the transversity GPDs. The first ever evaluation of this decomposition was demonstrated.
\end{abstract}

Keywords: Pseudoscalar; meson; electroproduction; Generalized Parton Distributions; Transversity.

PACS numbers: 13.60.Le, 14.20.Dh, 14.40.Be, 24.85.+p

\section{Introduction}

Understanding nucleon structure in terms of the fundamental degrees of freedom of Quantum Chromodynamics (QCD) is one of the main goals in the theory of strong interactions. In recent years it became clear that exclusive reactions may provide information about hadron structure encoded in so-called Generalized Parton Distributions $^{1,2}$ (GPDs). For each quark flavor $q$ there are eight GPDs. Four correspond to parton helicity-conserving (chiral-even) processes, denoted by $H^{q}, \tilde{H}^{q}, E^{q}$ and $\tilde{E}^{q}$, and four correspond to parton helicity-flip (chiral-odd) processes, ${ }^{3,4} H_{T}^{q}, \tilde{H}_{T}^{q}$,

This is an Open Access article published by World Scientific Publishing Company. It is distributed under the terms of the Creative Commons Attribution 3.0 (CC-BY) License. Further distribution of this work is permitted, provided the original work is properly cited. 
$E_{T}^{q}$ and $\tilde{E}_{T}^{q}$. The GPDs depend on three kinematic variables: $x, \xi$ and $t$. In a symmetric frame, $x$ is the average longitudinal momentum fraction of the struck parton before and after the hard interaction and $\xi$ (skewness) is half of the longitudinal momentum fraction transferred to the struck parton. The skewness can be expressed in terms of the Bjorken variable $x_{B}$ as $\xi \simeq x_{B} /\left(2-x_{B}\right)$. Here $x_{B}=Q^{2} /(2 p \cdot q)$ and $t=\left(p-p^{\prime}\right)^{2}$, where $p$ and $p^{\prime}$ are the initial and final four-momenta of the nucleon.

When the theoretical calculations for longitudinal virtual photons were compared with the JLab $\pi^{0}$ data ${ }^{5,6}$ they were found to underestimate the measured cross sections by more than an order of magnitude in their accessible kinematic regions. The failure to describe the experimental results with quark helicity-conserving operators stimulated a consideration of the role of the chiral-odd quark helicity-flip processes. Pseudoscalar meson electroproduction, and in particular $\pi^{0}$ production in the reaction $e p \rightarrow e^{\prime} p^{\prime} \pi^{0}$, was identified ${ }^{7-9}$ as especially sensitive to the quark helicityflip subprocesses. During the past few years, two parallel theoretical approaches $(\mathrm{GL})^{7,10}$ and $(\mathrm{GK})^{8,9}$ have been developed utilizing the chiral-odd GPDs in the calculation of pseudoscalar meson electroproduction. The GL and GK approaches, though employing different models of GPDs, lead to transverse photon amplitudes that are much larger than the longitudinal amplitudes.

\section{Definition of Structure Functions}

The unpolarized reduced meson cross section is described by 4 structure functions $\sigma_{T}, \sigma_{L}, \sigma_{T T}$ and $\sigma_{L T}$

$$
2 \pi \frac{d^{2} \sigma\left(\gamma^{*} p \rightarrow p \pi^{0}\right)}{d t d \phi_{\pi}}=\frac{d \sigma_{T}}{d t}+\epsilon \frac{d \sigma_{L}}{d t}+\epsilon \frac{d \sigma_{T T}}{d t} \cos 2 \phi+\sqrt{2 \epsilon(1+\epsilon)} \frac{d \sigma_{L T}}{d t} \cos \phi .
$$

References $^{9,10}$ obtain the following relations for unpolarized structure functions:

$$
\begin{gathered}
\frac{d \sigma_{L}}{d t}=\frac{4 \pi \alpha}{k^{\prime}} \frac{1}{Q^{4}}\left\{\left(1-\xi^{2}\right)|\langle\tilde{H}\rangle|^{2}-2 \xi^{2} \operatorname{Re}\left[\langle\tilde{H}\rangle^{*}\langle\tilde{E}\rangle\right]-\frac{t^{\prime}}{4 m^{2}} \xi^{2}|\langle\tilde{E}\rangle|^{2}\right\}, \\
\frac{d \sigma_{T}}{d t}=\frac{4 \pi \alpha}{2 k^{\prime} Q^{4}}\left[\left(1-\xi^{2}\right)\left|\left\langle H_{T}\right\rangle\right|^{2}-\frac{t^{\prime}}{8 m^{2}}\left|\left\langle\bar{E}_{T}\right\rangle\right|^{2}\right] \\
\frac{d \sigma_{L T}}{d t}=\frac{4 \pi \alpha}{\sqrt{2} k^{\prime} Q^{4}} \xi \sqrt{1-\xi^{2}} \frac{\sqrt{-t^{\prime}}}{2 m} \operatorname{Re}\left[\left\langle H_{T}\right\rangle^{*}\langle\tilde{E}\rangle\right] \\
\frac{d \sigma_{T T}}{d t}=\frac{4 \pi \alpha}{k^{\prime} Q^{4}} \frac{t^{\prime}}{16 m^{2}}\left|\left\langle\bar{E}_{T}\right\rangle\right|^{2}
\end{gathered}
$$

Here $m$ is the mass of the proton, $t^{\prime}=t-t_{\text {min }}$, where $\left|t_{\text {min }}\right|$ is the minimum value of $|t|$ corresponding to $\theta_{\pi}=0, k^{\prime}\left(Q^{2}, x_{B}\right)$ is a phase space factor and $\bar{E}_{T}=2 \widetilde{H}_{T}+E_{T}$. The brackets $\left\langle H_{T}\right\rangle$ and $\left\langle\bar{E}_{T}\right\rangle$ denote the convolution of the elementary process $\gamma^{*} q \rightarrow q \pi^{0}$ with the GPDs $H_{T}$ and $\bar{E}_{T}$. We call them generalized form factors. 


\section{Experimental Data}

Cross section of the reaction $e p \rightarrow e p \pi^{0}$ measured by the CLAS collaboration at Jlab at 1800 kinematic points in bins of $Q^{2}, x_{B}, t$ and $\phi$ were published in Refs. 5 and 6. Structure functions $\sigma_{U}=\sigma_{T}+\epsilon \sigma_{L}, \sigma_{L T}$ and $\sigma_{T T}$ have been obtained. These functions were compared with the predictions of the GPD models. ${ }^{9,10}$ CLAS confirmed that the measured unseparated cross sections are much larger than expected from leading-twist handbag calculations which are dominated by longitudinal photons. The same conclusion can be made in an almost model-independent way by noting that the structure functions $\sigma_{U}$ and $\left|\sigma_{T T}\right|$ are comparable to each other while $\left|\sigma_{L T}\right|$ is quite small (see Fig.1). Cross section and structure functions for $e p \rightarrow e \eta p$ were also obtained in parallel. The comparison of the $\pi^{0}$ and preliminary $\eta$ structure functions is shown in Fig. 1. $\sigma_{U}$ drops by a factor of 2.5 for $\eta$ in comparison with $\pi^{0}$ and $\sigma_{T T}$ drops by a factor of 10 . The GK GPD model ${ }^{9}$ (curves) follows the experimental data. The inclusion of $\eta$ data into consideration strengthens the statement about the transversity GPD dominance in the pseudoscalar electroproduction process.

\section{Generalized Form Factors}

The squared magnitudes of the generalized form factors $\left|\left\langle H_{T}\right\rangle\right|^{2}$ and $\left|\left\langle\bar{E}_{T}\right\rangle\right|^{2}$ may be directly extracted from the experimental data (see Eqs. 2 and 4 ) in the framework of GPD models.

$$
\begin{aligned}
\left|\left\langle\bar{E}_{T}\right\rangle^{\pi, \eta}\right|^{2} & =\frac{k^{\prime} Q^{4}}{4 \pi \alpha} \frac{16 m^{2}}{t^{\prime}} \frac{d \sigma_{T T}^{\pi, \eta}}{d t} \\
\left|\left\langle H_{T}\right\rangle^{\pi, \eta}\right|^{2} & =\frac{2 k^{\prime} Q^{4}}{4 \pi \alpha} \frac{1}{1-\xi^{2}}\left[\frac{d \sigma_{T}^{\pi, \eta}}{d t}+\frac{d \sigma_{T T}^{\pi, \eta}}{d t}\right] .
\end{aligned}
$$

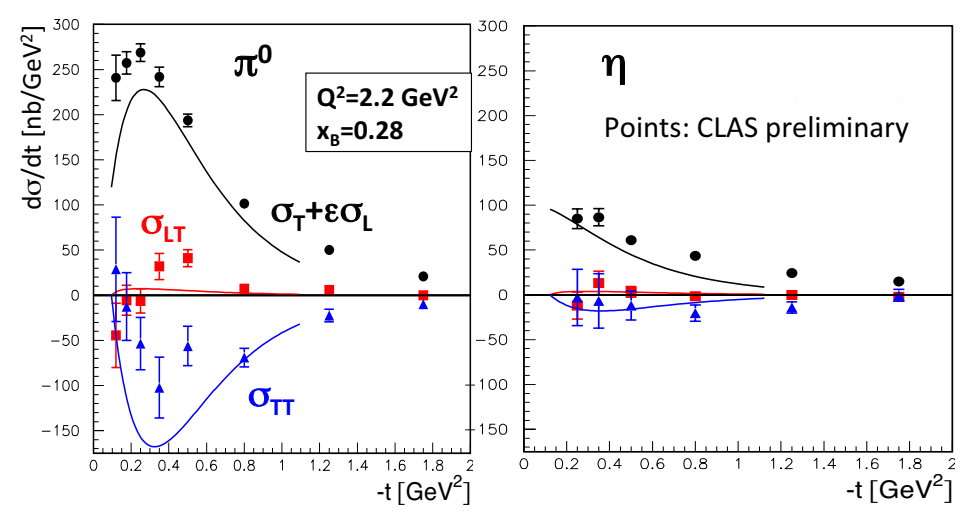

Fig. 1. (Color online) Structure functions $\sigma_{T}+\epsilon \sigma_{L}$ (black), $\sigma_{T T}$ (blue) and $\sigma_{L T}$ (red) as a function of $-t$ for $\pi^{0}$ (left) and $\eta$ (right) exclusive electroproduction for kinematic point $\left(Q^{2}=\right.$ $\left.2.2 \mathrm{GeV}^{2}, x_{B}=0.28\right)$. Data points: CLAS, preliminary. Curves: theoretical predictions produced with the GK handbag model. 

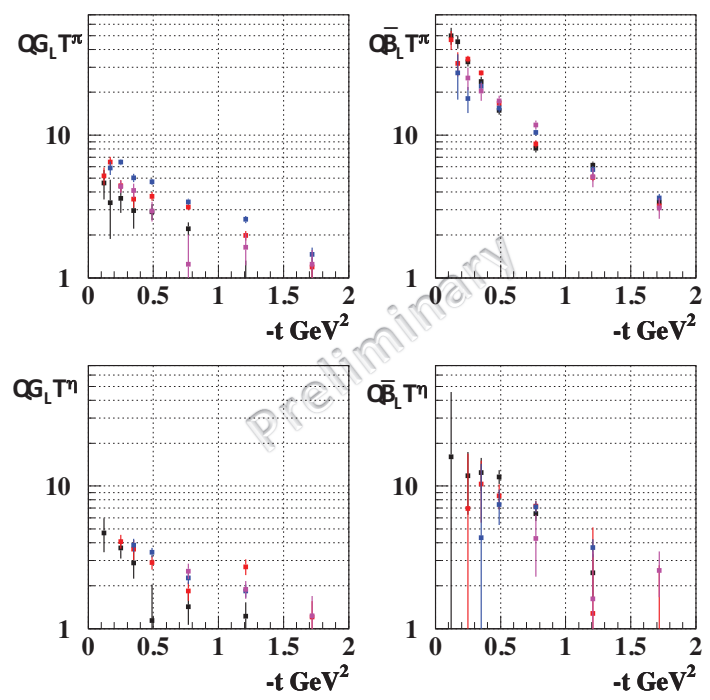

Fig. 2. (Color online) Data points: CLAS, preliminary. Top left: $\left|\left\langle H_{T}\right\rangle^{\pi}\right|$, top right: $\left|\left\langle\bar{E}_{T}\right\rangle^{\pi}\right|$, bottom left: $\left|\left\langle H_{T}\right\rangle^{\eta}\right|$, bottom right: $\left|\left\langle\bar{E}_{T}\right\rangle^{\eta}\right|$ as a function of $-t$ for different values of $\left(Q^{2}\left[G e V^{2}\right], x_{B}\right)=(1.2,0.15)$ black, $(1.8,0.22)$ red, $(2.2,0.27)$ blue, $(2.7,0.34)$ magenta.

Fig. 2 presents the modules of the generalized form factors $\left|\left\langle H_{T}\right\rangle^{\pi}\right|,\left|\left\langle\bar{E}_{T}\right\rangle^{\pi}\right|,\left|\left\langle H_{T}\right\rangle^{\eta}\right|$ and $\left|\left\langle\bar{E}_{T}\right\rangle^{\eta}\right|$ for 4 different kinematics. Note the dominance of the $\left|\left\langle\bar{E}_{T}\right\rangle\right|$ over $\left|\left\langle H_{T}\right\rangle\right|$ for both $\pi^{0}$ and $\eta$ for all kinematics.

\section{Flavor Decomposition}

In electroproduction the GPDs $F_{i}$ appears in the following combinations

$$
\begin{aligned}
& F_{i}^{\pi}=\frac{1}{\sqrt{2}}\left[e_{u} F_{i}^{u}-e_{d} F_{i}^{d}\right] \\
& F_{i}^{\eta}=\frac{1}{\sqrt{6}}\left[e_{u} F_{i}^{u}+e_{d} F_{i}^{d}-2 e_{s} F_{i}^{s}\right]
\end{aligned}
$$

The $q$ and $\bar{q}$ GPDs contribute in the quark combinations $F_{i}^{q}-F_{i}^{\bar{q}}$. Hence there is no contribution from the strange quarks if we assume that $F_{i}^{s} \simeq F_{i}^{\bar{s}}$. For flavor decomposition we have to take into account the decay constants $f_{\pi}$ and $f_{\eta}$, the chiral condensate constants $\mu_{\pi^{0}}=2.57 \mathrm{GeV}, \mu_{1}=0.958 \mathrm{GeV}$ and $\mu_{8}=2.32 \mathrm{GeV}$, and the contribution from singlet and octet $\eta$ states. $^{9}$

$$
F_{i}^{\eta}=F_{i}^{\pi}\left(\cos \theta_{8}-\sqrt{2} \frac{\mu_{1}}{\mu_{8}} \frac{f_{1}}{f_{8}} \sin \theta_{1}\right) \frac{f_{8}}{f_{\pi^{0}}} \frac{\mu_{8}}{\mu_{\pi^{0}}}=\frac{F_{i}^{8}}{k_{\eta}},
$$

where the mixing angles are: $\theta_{8}=-21.2^{\circ}$ and $\theta_{1}=-9.2^{\circ}$. The octet and singlet wave functions are very similar and the decay constants are close as well $f_{8}=1.26 f_{\pi}$ 
and $f_{1}=1.17 f_{\pi}$. The overall factor for the $\eta$ meson is $k_{\eta}=0.863$. Using $e_{u}=\frac{2}{3}$ and $e_{d}=-\frac{1}{3}$ we will end up with equations

$$
\begin{aligned}
F_{i}^{\pi} & =\frac{1}{3 \sqrt{2}}\left[2 F_{i}^{u}+F_{i}^{d}\right] \\
k_{\eta} F_{i}^{\eta} & =\frac{1}{3 \sqrt{6}}\left[2 F_{i}^{u}-F_{i}^{d}\right] .
\end{aligned}
$$

Experimentally we have access only to the $\left|\left\langle F_{i}^{\pi}\right\rangle\right|^{2}$ and $\left|\left\langle F_{i}^{\eta}\right\rangle\right|^{2}$ (see Eq. 5). The final equation for the $\left\langle H_{T}\right\rangle$ convolution reads

$$
\left\{\begin{array}{l}
\frac{1}{18}\left|2\left\langle H_{T}\right\rangle^{u}+\left\langle H_{T}\right\rangle^{d}\right|^{2}=\left|\left\langle H_{T}\right\rangle^{\pi}\right|^{2} \\
\frac{1}{54}\left|2\left\langle H_{T}\right\rangle^{u}-\left\langle H_{T}\right\rangle^{d}\right|^{2}=k_{\eta}^{2}\left|\left\langle H_{T}\right\rangle^{\eta}\right|^{2}
\end{array}\right.
$$

and simular equations for $\left\langle\bar{E}_{T}\right\rangle$.

The solution of these equations will lead to the flavor decomposition of the transversity GPDs $\left\langle H_{T}\right\rangle^{u}$ and $\left\langle H_{T}\right\rangle^{d}$ as well as $\left\langle\bar{E}_{T}\right\rangle^{u}$ and $\left\langle\bar{E}_{T}\right\rangle^{d}$. However the convolution integrals have real and imaginary parts. So it is impossible to solve these equations unambiguously with only two equations in hands. As a guidance we can estimate the form factors if we suppose that the relative phase $\Delta \phi$ between $\left\langle H_{T}\right\rangle^{u}$ and $\left\langle H_{T}\right\rangle^{d}$ equals 0 or 180 degrees. Ignoring an overall phase, the form factors are then real and we arbitrarily choose the solution with $\left\langle H_{T}\right\rangle^{u}$ and $\left\langle\bar{E}_{T}\right\rangle^{u}$ positive. Fig. 3 presents $\left\langle H_{T}\right\rangle^{u},\left\langle H_{T}\right\rangle^{d},\left\langle\bar{E}_{T}\right\rangle^{u}$ and $\left\langle\bar{E}_{T}\right\rangle^{d}$ for one kinematic point
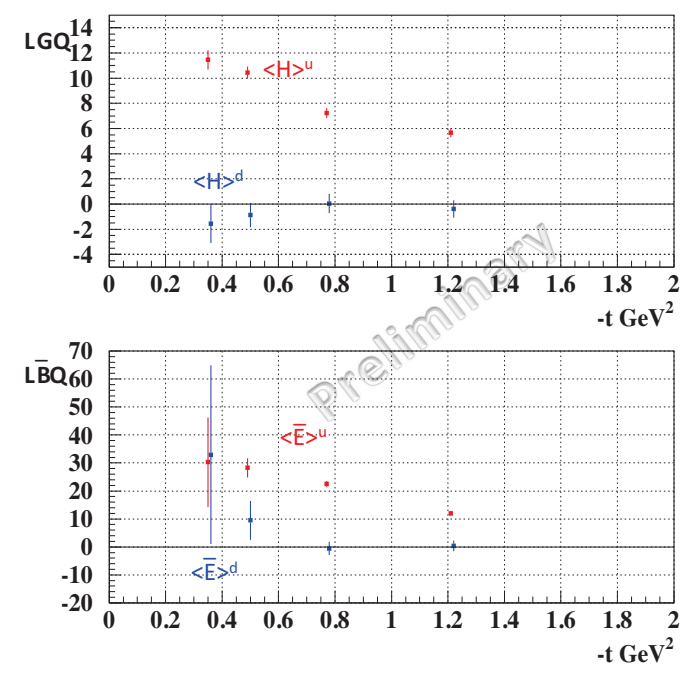

Fig. 3. (Color online) Preliminary. Top: Extracted $\left\langle H_{T}\right\rangle^{u}$ (red) and $\left\langle H_{T}\right\rangle^{d}$ (blue); Bottom: $\left\langle\bar{E}_{T}\right\rangle^{u}$ (red) and $\left\langle\bar{E}_{T}\right\rangle^{d}$ (blue), as a function of $-t$ for $Q^{2}=2.2 \mathrm{GeV}^{2}$ and $x_{B}=0.27$. 
$\left(Q^{2}=2.2 \mathrm{GeV}^{2}, x_{B}=0.27\right)$ calculated in this assumption. Note the different signs of $\left\langle H_{T}\right\rangle^{u}$ and $\left\langle H_{T}\right\rangle^{d}$ convolutions and the same sign of $\left\langle\bar{E}_{T}\right\rangle^{u}$ and $\left\langle\bar{E}_{T}\right\rangle^{d}$.

\section{Conclusion}

Differential cross sections of exclusive $\pi^{0}$ and $\eta$ electroproduction have been obtained in the few- $\mathrm{GeV}$ region at more than 1800 kinematic points in bins of $Q^{2}, x_{B}, t$ and $\phi_{\pi}$. Virtual photon structure functions $\sigma_{U}, \sigma_{T T}$ and $d \sigma_{L T}$ have been obtained. It is found that $\sigma_{U}$ and $\sigma_{T T}$ are comparable in magnitude with each other, while $\sigma_{L T}$ is very much smaller than either. Generalized form factors of the transversity GPDs $\left\langle H_{T}\right\rangle^{\pi, \eta}$ and $\left\langle\bar{E}_{T}\right\rangle^{\pi, \eta}$ were directly extracted from the experimental observables for the first time. It was found that the GPD $\bar{E}_{T}$ dominates in pseudoscalar meson production. The combined $\pi^{0}$ and $\eta$ data opens the way for the flavor decomposition of the transversity GPDs. Within some simplifying assumptions, the decomposition has been demonstrated for the first time.

\section{Acknowledgments}

The author thanks G. Goldstein, S. Goloskokov, P. Kroll, J. M. Laget, S. Liuti and A. Radyushkin for many informative discussions and making available the results of their calculations. This work was supported in part by the U.S. Department of Energy and National Science Foundation, The Jefferson Science Associates (JSA) operates the Thomas Jefferson National Accelerator Facility for the United States Department of Energy under contract DE-AC05-06OR23177.

\section{References}

1. X. Ji, Phys. Rev. Lett. 78, 610 (1997); Phys. Rev. D 55, 7114 (1997).

2. A.V. Radyushkin, Phys. Lett. B 380, 417 (1996); Phys. Rev. D 56, 5524 (1997).

3. P. Hoodbhoy and X. Ji, Phys. Rev. D 58, 054006 (1998).

4. M. Diehl, Phys. Rep. 388, 41 (2003) and references within.

5. I. Bedlinskiy et al. (CLAS Collaboration), Phys. Rev. Lett. 109, 112001 (2012).

6. I. Bedlinskiy et al. (CLAS Collaboration), Phys. Rev. C 90, 025205 (2014).

7. S. Ahmad, G. R. Goldstein and S. Liuti, Phys. Rev. D 79, 054014 (2009).

8. S. V. Goloskokov and P. Kroll, Eur. Phys. J. C 65, 137 (2010).

9. S. V. Goloskokov and P. Kroll, Eur. Phys. J. A 47, 112 (2011).

10. G. Goldstein, J. O. Gonzalez-Hernandez and S. Liuti, Phys. Rev. D 84, 034007 (2011); Int. J. Mod. Phys. Conf. Ser. 20, 222 (2012); J. Phys. G: Nucl. Part. Phys. 39115001 (2012). 